\title{
WEAKLY EQUICONTINUOUS SCHAUDER BASES
}

\author{
THURLOW A. COOK $^{1}$
}

Let $E$ be a locally convex Hausdorff linear topological vector space, henceforth called simply a locally convex space. A Schauder basis for $E$ is a sequence of vectors $\left\{x_{n}\right\}$ in $E$ together with a sequence $\left\{f_{n}\right\}$ of continuous linear functionals from $E^{*}$ such that $f_{k}\left(x_{j}\right)=\delta_{k, j}$ and for each $x \in E, x=\sum_{n=1}^{\infty} f_{n}(x) x_{n}$, convergence being in the topology of $E$. For each positive integer $m$ let $S_{m}(x)=\sum_{n=1}^{m} f_{n}(x) x_{n}$. We call the basis $\left(x_{n} ; f_{n}\right)$ equicontinuous when the partial sum operators $\left(S_{m}\right)$ are equicontinuous in the given topology of $E$.

In this note a necessary and sufficient condition for a Schauder basis to be weakly equicontinuous is given and complete locally convex spaces with weakly equicontinuous Schauder bases are characterized. A form of the latter result has been obtained in [1, p. 268]. Equicontinuous bases were introduced in [3, p. 208].

If the space $E$ is barrelled then by the Banach-Steinhaus Theorem, any Schauder basis of $E$ is equicontinuous. However, if $E$ is examined in its weak topology considerably different results occur.

1. Theorem. Let $E$ be a locally convex space with a Schauder basis $\left(x_{n} ; f_{n}\right)$. Then this basis is weakly equicontinuous if and only if $\left\{f_{n}\right\}$ is a Hamel basis for $E^{*}$. Moreover, if $E$ is complete, these conditions hold if and only if $E$ is linearly homeomorphic to (s), the countable product of reals.

Proof. Suppose $\left(x_{n} ; f_{n}\right)$ is weakly equicontinuous. It follows that if $f^{\prime} \in E^{*}$, then $\left\{f^{\prime} \circ S_{n}: n \in \omega\right\}$ is a family of weakly equicontinuous linear functions. It is well known (see e.g., $[2$, p. 161]) that $\left\{f^{\prime} \circ S_{n}: n \in \omega\right\}$ is thus finite dimensional. Consequently, $\left\{f_{n}\right\}$ is a Hamel basis for $E^{*}$, since $\left(f_{n}\right)$ is a $w^{*}$-Schauder basis for $E^{*}$. The converse is trivial.

To prove the second statement observe that all Schauder bases in (s) are weakly equicontinuous. This follows since the weak and Mackey topologies are the same.

Now suppose the conditions hold. The topology $w\left(E, E^{*}\right)$ is metrizable since $E^{*}$ has a countable Hamel basis [5, Theorem 6, p. 150]. By $[4$, Theorem 3.4, p. 132] this weak topology is the Mackey topology. Therefore $E$ is a Fréchet space.

Received by the editors December 7, 1968 and, in revised form, April 23, 1969.

1 Supported in part by the National Science Foundation Grant GP-5952. 
Define the following linear function $\phi$ of $E$ into $(s)$ : if $x \in E$ then let $\phi(x)=\sum_{n=1}^{\infty} f_{n}(x) e_{n}$ where $e_{n}$ is the $n$th coordinate unit vector in (s) and $x=\sum_{i=1}^{\infty} f_{i}(x) x_{i}$. The function $\phi$ is clearly linear. Also $\phi(E)$ $=(s)$. To see this let $\left\{a_{n}\right\} \in(s)$ and let $f \in E^{*}$; then for $k$ and $j$ large enough $f\left(\sum_{k}^{j} a_{n} x_{n}\right)=\sum_{k}^{j} a_{n} f\left(x_{n}\right)=0$ since $E^{*}$ has a countable Hamel basis. Hence $\left\{\sum_{n=1}^{k} a_{n} x_{n}: k \in \omega\right\}$ is a weakly hence Mackey Cauchy sequence and therefore convergent. Consequently, there is an $x \in E$ such that $x=\sum_{i=1}^{\infty} a_{i} x_{i}$ and $\phi(x)=\sum_{n=1}^{\infty} a_{n} e_{n}=\left\{a_{n}\right\}$. It follows from the Banach-Steinhaus theorem that $\phi$ is continuous.

By the open mapping theorem we have $E$ linearly homeomorphic to $(s)$.

\section{REFERENCES}

1. E. Dubinsky and J. R. Retherford, Schauder bases and Köthe sequence spaces, Trans. Amer. Math. Soc. 130 (1968), 265-280.

2. J. L. Kelley and I. Namioka, Linear topological spaces, Van Nostrand, Princeton, N. J., 1963.

3. C. W. McArthur and J. R. Retherford, Uniform and equicontinuous Schauder bases of subspaces, Canad. J. Math. 17 (1965), 207-212.

4. H. H. Schaefer, Topological vector spaces, Macmillan, New York, 1966.

5. A. Wilansky, Functional analysis, Blaisdell, Waltham, Mass., 1964.

UNIVERSity of MASSACHUSETTS 